Relations industrielles

Industrial Relations

\title{
The Catholic Movement of Employers and Managers. By Joseph B. Gremillon. Gregorian University Press, 4 Piazza della Pilotta, Rome, 1961, 217 pages.
}

\section{Gérard Dion}

Volume 17, numéro 1, janvier 1962

URI : https://id.erudit.org/iderudit/1021666ar

DOI : https://doi.org/10.7202/1021666ar

Aller au sommaire du numéro

Éditeur(s)

Département des relations industrielles de l’Université Laval

ISSN

0034-379X (imprimé)

1703-8138 (numérique)

Découvrir la revue

Citer ce compte rendu

Dion, G. (1962). Compte rendu de [The Catholic Movement of Employers and Managers. By Joseph B. Gremillon. Gregorian University Press, 4 Piazza della Pilotta, Rome, 1961, 217 pages.] Relations industrielles / Industrial Relations, 17(1), 93-94. https://doi.org/10.7202/1021666ar

Tous droits réservés (C Département des relations industrielles de l’Université Laval, 1962
Ce document est protégé par la loi sur le droit d'auteur. L'utilisation des services d’Érudit (y compris la reproduction) est assujettie à sa politique d'utilisation que vous pouvez consulter en ligne.

https://apropos.erudit.org/fr/usagers/politique-dutilisation/ 
- Les hôpitaux dans la Province de Québec \$, Département d'Action Sociale, Conférence Catholique Canadienne, Ottawa 1961, 103 pp.

Les problèmes relatifs à la protection de la santé constituent une des préoccupations actuelles les plus profondes aussi bien des gouvernants, que des mouvements ouvriers et des citoyens en général. L'assurance-santé donne un excellent exemple de cet état d'esprit: alors qu'il n'en était que vaguement question il a quelques années, elle fournit maintenant une source abondante de publicité électorale aux principaux partis politiques canadiens.

Or, dans ce vaste domaine, une mesure de sécurité sociale bien connue est déjà à l'épreuve dans toutes les provinces du pays: l'assurance-hospitalisation. Il est maintenant possible de se rendre compte de ses avantages, de ses inconvénients et de ses limitations. Elle suscite maintes discussions, surtout dans le Québec où elle n'a qu'un peu plus d'un an d'existence. On peut donc dire que la brochure cLes hôpitaux dans la Province de Québec s vient à point. Comme son nom lindique, elle touche les hôpitaux du Québec et renferme une impressionnante documentation sur les institutions hospitalières de la Province: confessionalité, année d'incorporation, genre, situation géographique, capacité, nom des directeurs, etc. C'est ainsi que le lecteur apprendra que, contrairement à la croyance populaire, la majorité des hôpitaux québecois $(64.5 \%)$ appartiennent à des laīcs; ceux-ci ne représentent toutefois que $\mathbf{4 1 . 5 \%}$ de la capacité totale en lits.

Cependant l'intérêt de cette brochure nous apparait résider moins dans son aspect statistique, bien que celui-ci soit très utile, que dans la collection de documents qu'elle présente sur le programme d'assurance-hospitalisation lui-même et sur certains événements historiques.

On ne peut en effet trouver à aucun autre endroit, colligés dans une même brochure, des textes comme ceux de la loi fédérale d'assurance-hospitalisation, des amendements à cette loi, de la loi provinciale de 1960 et de ses règlements. On peut aussi y consulter une copie du contrat hôpital-gouvernement, ainsi que deux mémoires soumis par des associations d'hôpitaux québecois à la Commis- sion Royale d'enquête sur l'assurancehospitalisation.

Dans la catégorie des documents historiques, on trouvera plusieurs déclarations officielles de l'épiscopat sur les problèmes hospitaliers et certains communiqués qui démontrent le souci longtemps manifesté par l'autorité religieuse de coordonner et d'unifier l'action des quatre grandes associations hospitalières confessionnelles du Québec. D'ailleurs une des sections de la brochure contient justement une précieuse documentation sur ces diverses associations.

La brochure \& Les hôpitaux dans la Province de Québec » s'avèrera certainement d'une grande utilité pour ceux qu'intéressent le problème hospitalier dans toutes ses dimensions: législation, unification des efforts, conformité par rapport aux besoins, etc. Cet ouvrage étant le premier de la série dite $<$ Hôpitaux $\gg$, il était logique qu'il contienne tous ces renseignements fondamentaux. Il serait à souhaiter que les autres qui lui succéderont s'attachent à des études de situations concrètes, à des analyacs de tendances et même à des exposés sur les problèmes financiers des institutions hospitalières. Si elle avait pu poursuivre son travail, la Commission Royale d'enquête sur l'assurance-hospitalisation aurait certainement livré au public québecois une intéressante documentation sur ces sujets; si on en juge par sa première publication sur les hôpitaux, le Département d'Action Sociale de la Conférence Catholique Canadienne semble devoir combler cette lacune.

Claude Morun

The Catholic Movement of Employers and Managers. By Joseph B. Gremillon. Gregorian University Press, 4 Piazza della Pilotta, Rome, 1961, 217 pages.

Ce volume est la publication d'une thèse préparée par l'auteur à l'Université Grégorienne de Rome sur l'Union internationale des associations patronales catholiques. (UNIAPAC)

Dans un court chapitre, l'auteur, qui est prêtre américain, présente ce groupement fondé officiellement en 1949, mais qui est la continuation d'une autre organisation mise sur pied en 1931. Il prend la peine de noter que le sujet de 
ReLATions IndUStruelies, vol. 17 , No 1

son étude n'est pas un fait accompli, une organisation statique, mais une institution en voie de développement selon les lignes d'une pensée, d'une doctrine qui impose aux employeurs chrétiens le devoir de se comporter en chrétiens dans leur vie économico-professionnelle.

Le second chapitre est consacré à la définition et à l'histoire de l'UNIAPAC. L'auteur montre comment graduellement les employeurs catholiques se sont groupés dans différents pays d'Europe (Hollande, Belgique, France) ont senti le besoin d'organiser des conférences internationales et ont essaimé tant au Canada qu'en Amérique latine. C'est surtout après la deuxième grande guerre que le mouvement a pris de la consistance.

Les chapitres suivants expliquent l'organisation du groupement, ses buts et politiques, son rôle éducatif et sa doctrine, ses méthodes d'action. L'auteur termine en faisant une évaluation de ce qu'a accompli l'UNIAPAC. En appendice, il donne une longue bibliographie des publications dont il s'est servi pour préparer cette thèse.

Nous avons lu cet ouvrage avec d'autant plus d'intérêt que nous avons été mêlé autrefois au développement du groupement canadien, l'Association professionnelle des industriels, qui est affilié à l'UNIAPAC. S'il nous faut regretter que l'auteur se soit un peu trop fié à des publications qui avaient un caractère beaucoup plus de propagande que des visées scientifiques, (c'est une question de détail), nous considérons qu'il a très bien réussi à saisir et à exprimer l'essentiel de l'UNIAPAC. C'est un mouvement qui cherche à former les personnes exercant la fonction patronale à la lumière de la doctrine sociale de l'Eglise et qui les incite à prendre ainsi leurs responsabilités dans leur vie professionnelle. Loin d'être un substitut des syndicats patronaux ou des associations professionnelles, ce mouvement prépare ses membres à y jouer un rôle positif et dynamique.

Cet ouvrage est le premier du genre en la matière. Il est une excellente synthèse qui permettra de bien comprendre la nature et le rôle de l'UNIAPAC et des groupements qui lui sont affiliés. Nous sommes assuré qu'il contribuera à éclaircir certains concepts et favorisera le développement de l'organisation chez ceux qui exercent la fonction patronale.

GÉrard Dron

Le profut comptable: fiction ou réalité? par Alphonse Riverin. Presses de l'Université Laval, Québec. 1961. 139 pages.

Les ouvrages qui remettent en question nos méthodes de travail et de pensée sont toujours fort intéressants. Malheureusement, si la production d'oeuvres de ce genre est peu considérable dans la plupart des disciplines, elle est, chez nous, presque inexistante dans le domaine des sciences de gestion. Il convient donc de saluer avec empressement la parution de cet ouvrage.

L'auteur y cherche comment les techniques comptables, si bien rodées par l'usage, pourraient emprunter à la science économique certains concepts qui, de leur côté, ont l'avantage de s'insérer dans une structure théorique rigide. Les deux disciplines y trouveraient leur profit et surtout la comptabilité pourrait devenir un outil bien plus utile qu'elle ne l'est présentement.

L'étude s'ouvre sur une discussion du concept de profit. L'auteur rejette tour à tour comme inutilisables plusieurs définitions proposées par des économistes: un concept ne peut être utile en ce domaine, s'il ne se prête à certaines mesures quantitatives. En définitive, il retient la célèbre définition de Hicks, à savoir que le profit d'une période est mesuré par la somme maximale qu'un individu peut dépenser sans entamer son capital. Une fois la définition établie, le professeur Riverin fait l'examen des postulats fondamentaux de la comptabilité. La critique du postulat de réalisation, suivie d'une intégration de la théorie des coûts, constitue sans doute une des meilleures parties de l'ouvrage. La notion de coût marginal permet à l'auteur d'expliquer le fonctionnement de la méthode des coûts variables. (Cette technique d'accumulation des coûts est sans doute mieux connue sous le nom de « direct costing »). Toutes les explications relatives à cette méthode sont illustrées par l'exemple de la page 62. Le lecteur regrettera que la présentation de ce tableau en rende l'étude plus difficile que nécessaire. En effet, les sous-titres suggèrent d'abord 